\title{
SOME INEQUALITIES OF ALGEBRAIC POLYNOMIALS WITH NONNEGATIVE COEFFICIENTS
}

\author{
WEIYU CHEN
}

ABSTRACT. Let $S_{n}$ be the collection of all algebraic polynomials of degree $\leq n$ with nonnegative coefficients. In this paper we discuss the extremal problem

$$
\sup _{p_{n}(x) \in S_{n}} \frac{\int_{a}^{b}\left(p_{n}^{\prime}(x)\right)^{2} \omega(x) d x}{\int_{a}^{b} p_{n}^{2}(x) \omega(x) d x}
$$

where $\omega(x)$ is a positive and integrable function. This problem is solved completely in the cases

(i) $[a, b]=[-1,1], \omega(x)=\left(1-x^{2}\right)^{\alpha}, \alpha>-1$;

(ii) $[a, b)=[0, \infty), \omega(x)=x^{\alpha} e^{-x}, \alpha>-1$;

(iii) $(a, b)=(-\infty, \infty), \omega(x)=e^{-\alpha x^{2}}, \alpha>0$.

The second case was solved by Varma for some values of $\alpha$ and by Milovanovic completely. We provide a new proof here in this case.

\section{INTRODUCTION}

In this paper we investigate the following extremal problem

$$
\sup _{p_{n}(x) \in S_{n}} \frac{\int_{a}^{b}\left(p_{n}^{\prime}(x)\right)^{2} \omega(x) d x}{\int_{a}^{b} p_{n}^{2}(x) \omega(x) d x}
$$

where

$$
S_{n}=\left\{p_{n}(x): p_{n}(x)=\sum_{i=0}^{n} a_{i} x^{i}, a_{i} \geq 0,0 \leq i \leq n\right\},
$$

and $\omega(x):(a, b) \rightarrow R$ is a positive and integrable function.

In the case $[a, b)=[0, \infty), \omega(x)=x^{\alpha} e^{-x}, \alpha>-1$, the extremal problem (1) was initiated and solved by Varma [10] in the cases $0 \leq \alpha \leq 1 / 2$ and $(\sqrt{5}-1) / 2 \leq \alpha<\infty$. Later, it was solved completely by Milovanovic [4] for $-1<\alpha<\infty$.

In this note we consider the above extremal problem (1) for different weight functions on different intervals. Throughout this paper, we denote $S_{n}$ the collection of all algebraic polynomials of degree $\leq n$ with nonnegative coefficients. In Section 2, we provide the complete answer to the case $[a, b]=[-1,1]$, $\omega(x)=\left(1-x^{2}\right)^{\alpha}, \alpha>-1$. In the case $\alpha=0$, this result is an analogue of a

Received by the editors November 24,1993; originally communicated to the Proceedings of the $A M S$ by Andrew Bruckner.

1991 Mathematics Subject Classification. Primary 41A17; Secondary 26C05.

Key words and phrases. Markov inequality, nonnegative coefficients. 
theorem of Lorentz [3] in the $L_{\infty}$ norm. Indeed, that theorem holds for a wider class (Lorentz class) of polynomials, which was studied extensively by Scheick [7]. For some subsets of Lorentz class of polynomials, the extremal problem (1) was discussed by Milovanović and Petković [5] for the Jacobi weight.

In Section 3, we give a new proof of Milovanović's Theorem [4]. In our last section, Section 4 , we consider the weight function $\omega(x)=e^{-\alpha x^{2}}, \alpha>0$, on the interval $(-\infty, \infty)$.

The corresponding extremal problem for the unrestricted polynomials was discussed in Dörfler [1], [2], Mirsky [6] and Turán [8], which are Markov type inequalities in $L_{2}$ norm.

\section{THE WEIGHT $\omega(x)=\left(1-x^{2}\right)^{\alpha}$}

In this section, we discuss the extremal problem in the $L_{2}$ norm under the weight function $\omega(x)=\left(1-x^{2}\right)^{\alpha}, \alpha>-1$, on $[-1,1]$. For some special values of $\alpha$, we obtain several corollaries corresponding to some classic weight functions. The main result in this section is the following theorem.

Theorem 2.1. Let $p_{n}(x) \in S_{n}, \alpha>-1$; then

$$
\int_{-1}^{1}\left(p_{n}^{\prime}(x)\right)^{2}\left(1-x^{2}\right)^{\alpha} d x \leq \frac{2 n+2 \alpha+1}{2 n-1} n^{2} \int_{-1}^{1} p_{n}^{2}(x)\left(1-x^{2}\right)^{\alpha} d x
$$

with equality when $p_{n}(x)=x^{n}$.

Proof. Since $p_{n}(x) \in S_{n}$, we can write

$$
p_{n}(x)=\sum_{i=0}^{n} a_{i} x^{i}
$$

with $a_{i} \geq 0,0 \leq i \leq n$. Then

$$
p_{n}^{\prime}(x)=\sum_{i=1}^{n} i a_{i} x^{i-1}
$$

and

$$
\begin{aligned}
\int_{-1}^{1} p_{n}^{2}(x)\left(1-x^{2}\right)^{\alpha} d x & =\sum_{i=0}^{n} \sum_{j=0}^{n} a_{i} a_{j} \int_{-1}^{1} x^{i+j}\left(1-x^{2}\right)^{\alpha} d x \\
\int_{-1}^{1}\left(p_{n}^{\prime}(x)\right)^{2}\left(1-x^{2}\right)^{\alpha} d x & =\sum_{i=0}^{n} \sum_{j=0}^{n} a_{i} a_{j} i j \int_{-1}^{1} x^{i+j-2}\left(1-x^{2}\right)^{\alpha} d x
\end{aligned}
$$

Let

$$
\begin{aligned}
b_{i j} & =\int_{-1}^{1} x^{i+j}\left(1-x^{2}\right)^{\alpha} d x \\
& =\frac{1-(-1)^{i+j+1}}{2} B\left(\frac{i+j+1}{2}, \alpha+1\right)
\end{aligned}
$$

where $B(x, y)$ is the Beta function and

$$
\begin{aligned}
c_{i j} & =i j \int_{-1}^{1} x^{i+j-2}\left(1-x^{2}\right)^{\alpha} d x \\
& =i j \frac{1-(-1)^{i+j+1}}{2} B\left(\frac{i+j-1}{2}, \alpha+1\right)
\end{aligned}
$$


for $1 \leq i, j \leq n, c_{i j}=0$ if $i=0$ or $j=0$. Now denote

$$
B=\left(b_{i j}\right)_{0 \leq i, j \leq n}, \quad C=\left(c_{i j}\right)_{0 \leq i, j \leq n},
$$

and

$$
a=\left(a_{0}, a_{1}, \ldots, a_{n}\right)^{\top}
$$

then we can derive that

$$
\begin{gathered}
\int_{-1}^{1} p_{n}^{2}(x)\left(1-x^{2}\right)^{\alpha} d x=\sum_{i=0}^{n} \sum_{j=0}^{n} a_{i} a_{j} b_{i j}=a^{\top} B a, \\
\int_{-1}^{1}\left(p_{n}^{\prime}(x)\right)^{2}\left(1-x^{2}\right)^{\alpha} d x=\sum_{i=0}^{n} \sum_{j=0}^{n} a_{i} a_{j} c_{i j}=a^{\top} C a .
\end{gathered}
$$

Now it suffices to consider the following extremal problem:

$$
\sup _{a \in R_{+}^{n+1}} \frac{a^{\top} C a}{a^{\top} B a}
$$

where $R_{+}^{n+1}=\left\{a: a=\left(a_{0}, a_{1}, \ldots, a_{n}\right)^{\top}, a_{i} \geq 0,0 \leq i \leq n\right\}$. Or find the least $\lambda$ such that

$$
\frac{a^{\top} C a}{a^{\top} B a} \leq \lambda, \quad \text { for all } a \in R_{+}^{n+1},
$$

which is

$$
a^{\top}(\lambda B-C) a \geq 0, \quad \text { for all } a \in R_{+}^{n+1} .
$$

Observe that $b_{i j} \geq 0, c_{i j} \geq 0,0 \leq i, j \leq n$. If we can find a smallest $\lambda$ such that all the elements of $\lambda B-C$ are nonnegative, then we obtain (4) automatically. Notice also that the matrices $B$ and $C$ have the same structure; thus it suffices to find $\lambda$ such that

$$
\lambda b_{i j}-c_{i j} \geq 0, \quad \text { when } b_{i j} \neq 0,
$$

i.e.,

$$
\lambda \geq \frac{c_{i j}}{b_{i j}}=\frac{i j(i+j+2 \alpha+1)}{i+j-1}, \quad 1 \leq i, j \leq n .
$$

If we consider $c_{i j} / b_{i j}$ as a function of two continuous variables $i$ and $j$, then we have

$$
\frac{\partial}{\partial i}\left(\frac{i j(i+j+2 \alpha+1)}{i+j-1}\right)=\frac{j\left[i^{2}+(j-1)(2 i+j+2 \alpha+1)\right]}{(i+j-1)^{2}} \geq 0
$$

and similarly

$$
\frac{\partial}{\partial j}\left(\frac{i j(i+j+2 \alpha+1)}{i+j-1}\right)=\frac{i\left[j^{2}+(i-1)(2 j+i+2 \alpha+1)\right]}{(i+j-1)^{2}} \geq 0 ;
$$

thus this is ancreasing function of $i$ and $j$, and we can pick up

$$
\lambda=\left.\frac{i j(i+j+2 \alpha+1)}{i+j-1}\right|_{i=n, j=n}=\frac{2 n+2 \alpha+1}{2 n-1} n^{2} .
$$

To see that $\lambda$ is the best one, we can consider $p_{n}(x)=x^{n}$ or $a^{\top}=(0,0, \ldots$, $0,1)$. This completes the proof of the theorem.

For some special values of $\alpha$, we have the following corollaries. 
Corollary 2.2. Let $p_{n}(x) \in S_{n}$; then

$$
\int_{-1}^{1}\left(p_{n}^{\prime}(x)\right)^{2} d x \leq \frac{2 n+1}{2 n-1} n^{2} \int_{-1}^{1} p_{n}^{2}(x) d x
$$

with equality when $p_{n}(x)=x^{n}$.

Corollary 2.3. Let $p_{n}(x) \in S_{n}$; then

$$
\int_{-1}^{1}\left(p_{n}^{\prime}(x)\right)^{2}\left(1-x^{2}\right)^{-1 / 2} d x \leq \frac{2 n}{2 n-1} n^{2} \int_{-1}^{1} p_{n}^{2}(x)\left(1-x^{2}\right)^{-1 / 2} d x
$$

with equality when $p_{n}(x)=x^{n}$.

Corollary 2.4. Let $p_{n}(x) \in S_{n}$; then

$$
\int_{-1}^{1}\left(p_{n}^{\prime}(x)\right)^{2}\left(1-x^{2}\right)^{-1 / 2} d x \leq \frac{2 n+2}{2 n-1} n^{2} \int_{-1}^{1} p_{n}^{2}(x)\left(1-x^{2}\right)^{-1 / 2} d x
$$

with equality when $p_{n}(x)=x^{n}$.

In the case $\alpha=1$, a similar result was proved by Varma [9] for polynomials having real roots.

\section{The WeIGHT $\omega(x)=x^{\alpha} e^{-x}$}

We give a new proof of Milovanović's Theorem [4] in this section. Indeed we use the same argument as was used in the proof of Theorem 2.1. This time, we consider the weight function $\omega(x)=x^{\alpha} e^{-x}, \alpha>-1$, on the interval $[0, \infty)$.

Theorem 3.1. Let $p_{n}(x) \in S_{n}, \alpha>-1$; then

$$
\int_{0}^{\infty}\left(p_{n}^{\prime}(x)\right)^{2} x^{\alpha} e^{-x} d x \leq C_{n}(\alpha) \int_{0}^{\infty} p_{n}^{2}(x) x^{\alpha} e^{-x} d x
$$

where

and

$$
C_{n}(\alpha)= \begin{cases}1 /[(2+\alpha)(1+\alpha)], & -1<\alpha \leq \alpha_{n}, \\ n^{2} /[(2 n+\alpha)(2 n+\alpha-1)], & \alpha_{n} \leq \alpha<\infty\end{cases}
$$

$$
\alpha_{n}=\frac{1}{2}(n+1)^{-1}\left[\left(17 n^{2}+2 n+1\right)^{1 / 2}-3 n+1\right] .
$$

Moreover, $C_{n}(\alpha)$ is the best possible constant.

Proof. Let $p_{n}(x)=\sum_{i=0}^{n} a_{i} x^{i}, a_{i} \geq 0,0 \leq i \leq n$, then

$$
\begin{aligned}
\int_{0}^{\infty} p_{n}^{2}(x) x^{\alpha} e^{-x} d x & =\sum_{i=0}^{n} \sum_{j=0}^{n} a_{i} a_{j} \int_{0}^{\infty} x^{i+j+\alpha} e^{-x} d x \\
& =\sum_{i=0}^{n} \sum_{j=0}^{n} a_{i} a_{j} b_{i j}=a^{\top} B a
\end{aligned}
$$

where

$$
\begin{gathered}
b_{i j}=\int_{0}^{\infty} x^{i+j+\alpha} e^{-x} d x=\Gamma(i+j+\alpha+1), \\
B=\left(b_{i j}\right)_{0 \leq i, j \leq n} .
\end{gathered}
$$


And similarly, we have

$$
\int_{0}^{\infty}\left(p_{n}^{\prime}(x)\right)^{2} x^{\alpha} e^{-x} d x=\sum_{i=0}^{n} \sum_{j=0}^{n} a_{i} a_{j} c_{i j}=a^{\top} C a
$$

where

$$
\begin{aligned}
& c_{i j}= \begin{cases}i j \Gamma(i+j+\alpha-1), & 1 \leq i, j \leq n, \\
0, & i=0 \text { or } j=0,\end{cases} \\
& C=\left(c_{i j}\right)_{0 \leq i, j \leq n} .
\end{aligned}
$$

Therefore, we need to find the least $\lambda$ such that

$$
\lambda b_{i j}-c_{i j} \geq 0, \quad \text { for } 1 \leq i, j \leq n .
$$

That is, the maximum value of the function

$$
f(i, j):=\frac{c_{i j}}{b_{i j}}=\frac{i j}{(i+j+\alpha)(i+j+\alpha-1)} .
$$

Let $k=i+j$; then

$$
\begin{aligned}
f(i, j) & =\frac{i j}{(i+j+\alpha)(i+j+\alpha-1)} \\
& =\frac{i(k-i)}{(k+\alpha)(k+\alpha-1)}=: g(i, k) .
\end{aligned}
$$

If we consider $g$ as a function of two continuous variables $i$ and $k$, then we have

$$
\frac{\partial g(i, k)}{\partial i}=\frac{k-2 i}{(k+\alpha)(k+\alpha-1)} .
$$

Therefore, $g(i, k)$ takes on its maximum value at $i=k / 2$ if we fix $k$ (consider it as a function of $i$ alone). Now it suffices to consider the maximum value of the function

$$
h(k):=g\left(\frac{k}{2}, k\right)=\frac{k^{2}}{4(k+\alpha)(k+\alpha-1)} .
$$

Following the exactly same argument of Milovanovic [4, p. 425], we can see that the best possible value of $\lambda$ is $C_{n}(\alpha)$. We omit the details. This completes the proof.

Remark. The same idea also seems to work for other $L_{p}$ norms when $p$ is an integer, but they become more and more complicated as $p$ is bigger and bigger. We will not formulate them here. However, for the $L_{1}$ norm, the result is simple.

Theorem 3.2. Let $p_{n}(x) \in S_{n}, \alpha>-1$; then

$$
\int_{0}^{\infty} p_{n}^{\prime}(x) x^{\alpha} e^{-x} d x \leq \lambda_{n}(\alpha) \int_{0}^{\infty} p_{n}(x) x^{\alpha} e^{-x} d x
$$

where

$$
\lambda_{n}(\alpha)= \begin{cases}1 /(1+\alpha), & -1<\alpha \leq 0, \\ n /(n+\alpha), & 0 \leq \alpha<\infty\end{cases}
$$

Moreover, $\lambda_{n}(\alpha)$ is the best possible constant. 


\section{The WEIGHT $\omega(x)=e^{-\alpha x^{2}}$}

In this section we discuss the weight function $\omega(x)=e^{-\alpha x^{2}}, \alpha>0$, on the whole real line. The corresponding result is the following theorem.

Theorem 4.1. Let $p_{n}(x) \in S_{n}, \alpha>0$; then

$$
\int_{-\infty}^{\infty}\left(p_{n}^{\prime}(x)\right)^{2} e^{-\alpha x^{2}} d x \leq \frac{2 \alpha}{2 n-1} n^{2} \int_{-\infty}^{\infty} p_{n}^{2}(x) e^{-\alpha x^{2}} d x
$$

with equality when $p_{n}(x)=x^{n}$.

Proof. Let $p_{n}(x)=\sum_{i=0}^{n} a_{i} x^{i} \in S_{n}$; then

$$
\int_{-\infty}^{\infty} p_{n}^{2}(x) e^{-\alpha x^{2}} d x=\sum_{i=0}^{n} \sum_{j=0}^{n} a_{i} a_{j} b_{i j}=a^{\top} B a
$$

where

$$
\begin{gathered}
b_{i j}=\int_{-\infty}^{\infty} x^{i+j} e^{-\alpha x^{2}} d x \\
=\left(1-(-1)^{i+j+1}\right)(i+j-1) ! ! 2^{-(i+j) / 2-1} \alpha^{-(i+j+1) / 2} \sqrt{\pi}, \\
B=\left(b_{i j}\right)_{0 \leq i, j \leq n},
\end{gathered}
$$

and

$$
\int_{-\infty}^{\infty}\left(p_{n}^{\prime}(x)\right)^{2} e^{-\alpha x^{2}} d x=\sum_{i=0}^{n} \sum_{j=0}^{n} a_{i} a_{j} c_{i j}=a^{\top} C a
$$

where

$$
\begin{gathered}
c_{i j}=i j \int_{-\infty}^{\infty} x^{i+j-2} e^{-\alpha x^{2}} d x \\
=\left(1-(-1)^{i+j+1}\right) i j(i+j-3) ! ! 2^{-(i+j) / 2} \alpha^{-(i+j-1) / 2} \sqrt{\pi}, \\
C=\left(c_{i j}\right)_{0 \leq i, j \leq n .}
\end{gathered}
$$

For $i+j$ even, let

$$
f(i, j):=\frac{c_{i j}}{b_{i j}}=2 \alpha \frac{i j}{i+j-1}, \quad 1 \leq i, j \leq n ;
$$

then considering $f$ as a function of two continuous variables $i$ and $j$, we can obtain

$$
\frac{\partial f(i, j)}{\partial i}=\frac{2 \alpha j(j-1)}{(i+j-1)^{2}} \geq 0 \text { for } 1 \leq i, j \leq n,
$$

and

$$
\frac{\partial f(i, j)}{\partial j}=\frac{2 \alpha i(i-1)}{(i+j-1)^{2}} \geq 0, \quad \text { for } 1 \leq i, j \leq n .
$$

Therefore, $f(i, j)$ attains its maximum value at $i=n, j=n$, which implies the desired result.

Added in Proof. After this manuscript was written, the author learned that Professor A. K. Varma [11] had written a paper on the same subject. There are some overlaps between his results and our results in $\S \S 2$ and 3 , but we do use different methods. 


\section{REFERENCES}

1. P. Dörfler, New inequalities of Markov type, SIAM J. Math. Anal. 18 (1987), 490-494.

2. _ An extremal problem concerning a Markov-type inequality, SIAM J. Math. Anal. 22 (1991), 792-795.

3. G. G. Lorentz, Degree of approximation by polynomials with positive coefficients, Math. Ann. 151 (1963), 239-251.

4. G. V. Milovanović, An extremal problem for polynomials with nonnegative coefficients, Proc. Amer. Math. Soc. 94 (1985), 423-426.

5. G. V. Milovanović and M. S. Petković, Extremal problems for Lorentz classes of nonnegative polynomials in $L^{2}$ metric with Jacobi weight, Proc. Amer. Math. Soc. 102 (1988), 283-289.

6. L. Mirsky, An inequality of the Markov-Bernstein type for polynomials, SIAM J. Math. Anal. 14 (1983), 1004-1008.

7. J. T. Scheick, Inequalities for derivatives of polynomials of special type, J. Approx. Theory 6 (1972), 354-358.

8. P. Turán, Remark on a theorem of Erhard Schmidt, Mathematica 2 (1960), 373-378.

9. A. K. Varma, Some inequalities of algebraic polynomials having real zeros, Proc. Amer. Math. Soc. 75 (1979), 243-250.

10. _ Derivatives of polynomials with positive coefficients, Proc. Amer. Math. Soc. 83 (1981), 107-112.

11. __ Some inequalities of algebraic polynomials, preprint.

Department of Mathematics, University of Alberta, Edmonton, Alberta, Canada T6G 2G1 